\title{
Nanomateriales en las instituciones de educación superior y de investigación en México
}

\author{
Gregorio H. Cocoletzi, * R. A. Vázquez-Nava, ** \\ NoBORU TAKEUCHI***
}

\section{INTRODUCCIÓN}

La obtención o fabricación de materiales para usos cada vez más específicos ha sido fundamental para el avance de la humanidad a través de los tiempos. Tan es así que diferentes épocas históricas del desarrollo humano se clasifican de acuerdo con el avance del hombre en el control de los materiales: se habla, por ejemplo, de la edad de piedra, de la de bronce o la del hierro. El siglo pasado se caracterizó por un gran avance en la industria semiconductora o del silicio, lo que permitió la fabricación de sofisticados dispositivos electrónicos cada vez más pequeños. La necesidad de fabricar nuevos materiales con funcionalidades específicas ha hecho que hoy se pueda ver, medir, modificar y manipular átomos y moléculas. La nanociencia y la nanotecnología se ocupan del estudio y aplicación de sistemas de dimensiones muy pequeñas (entre 1-100 nanómetros). A estos tamaños los materiales (llamados nanomateriales) tienen un área superficial muy elevada con respecto a su volumen, y sus propiedades fundamentales (como color, dureza, punto de fusión, propiedades magnéticas, etc.) son diferentes con respecto a las que presentan en las escalas micro o macroscópicas. De tal forma, el confinamiento de la luz o de los electrones en dimensiones reducidas hace que las propiedades ópticas y electrónicas cambien.

La nanotecnología, al controlar la forma en cómo se acomodan los átomos y las moléculas, lo que busca es usar las propiedades de los nanomateriales en aplicaciones novedosas. Esta disciplina constituye una revolución cientificotecnológica, pues ha venido a transformar radicalmente los conocimientos, conceptos de manufactura y diseño de estos nuevos materiales.

Actualmente, los nanomateriales se están utilizando en un buen número de industrias para aplicaciones en sistemas electrónicos, magnéticos, optoelectrónicos, energéticos, catalíticos, biomédicos, farmacéuticos, y cosméticos.

En el año 2000, el entonces presidente de los Estados Unidos, William Clinton, impulsó un programa para desarrollar la nanociencia y la nanotecnología. Este proyecto permitió a la Unión Americana establecer de manera firme los proyectos de investigación para estudiar sistemas a escala nanométrica, como nanopartículas de metales o de semiconductores. Sin embargo, anterior a este programa, en la década de los 70's ya se estudiaban sistemas como pozos cuánticos y películas delgadas de semiconduc-

\footnotetext{
* Instituto de Física, Universidad Autónoma de Puebla, Apartado Postal J-48, Puebla 72570, México.

** Centro de Investigación en Óptica, León, Guanajuato, México.

*** Centro de Nanociencias y Nanotecnología, Universidad Nacional Autónoma de México, Ensenada, Baja California. Con el apoyo de la DGAPA: proyecto INI01809.
} 
tores y de metales donde los tamaños de los espesores eran de sólo varios nanómetros. La construcción de estos sistemas es posible gracias a las técnicas de crecimiento como la epitaxia de haces moleculares (MBE) y los métodos de deposición química de vapor. En las siguientes líneas daremos una breve explicación de su funcionamiento.

La epitaxia por haces moleculares (MBE del inglés molecular beam epitaxy) es un método de fabricación de películas delgadas y heteroestructuras de semiconductores, con el cual se alcanza una gran perfección cristalina. Consiste en la interacción de uno o más haces moleculares o atómicos con una superficie de un sustrato cristalino, la cual es calentada, resultando en un crecimiento capa por capa. Los haces moleculares se obtienen a partir de los elementos en estado sólido, los cuales se colocan en celdas de efusión. En este método es necesario tener un control perfecto de la temperatura y el vacío en la cámara de crecimiento. Un método similar al de MBE es el crecimiento por ablación de láser (PLD del inglés pulsed laser deposition), en el cual, un láser pulsado de alta intensidad se incide sobre un blanco (target), vaporizándolo y depositando una película delgada sobre el sustrato.

Otro método de fabricación de películas delgadas es el de deposición química de vapor o CVD (de sus siglas en inglés chemical vapor deposition). Es un proceso químico, en el que el sustrato es expuesto a uno o más precursores volátiles que reaccionan o se descomponen en la superficie del sustrato para producir el depósito deseado. Dependiendo de las condiciones específicas se tienen variantes diferentes del método. La deposición química organometálica de vapor (MOCVD del inglés metallic organometallic chemical vapor deposition) es un método de deposición química de vapor con el que se produce un crecimiento epitaxial de compuestos semiconductores resultantes de una reacción en la superficie de compuestos orgánicos y metalorgánicos. Tiene otros nombres tales como: epitaxia organometálica en fase de vapor (OMVPE), y deposición química organometálica en fase de vapor (OMCVD). Este método es muy usado para fabricar semiconductores III-V como el nitruro de aluminio o el arseniuro de galio.

En la deposición química de vapor asistida por plasma PECVD (del inglés plasma enhaced chemical vapor deposition) y RECVD (del inglés remote plasma enhaced chemical vapor deposition), también se depositan películas delgadas sobre un sustrato. Aquí, las reacciones químicas involucradas en el proceso ocurren después de la creación de un plasma de los gases reactantes. La ventaja de este método es que el depósito se hace muy rápido y a bajas temperaturas.

Un método similar al CVD es el depósito por capas atómicas (ALD del inglés atomic layer deposition), que se basa en el uso secuencial de un proceso químico en fase gaseosa. La mayoría de las reacciones ALD utilizan dos productos químicos, generalmente llamados precursores. Estos precursores reaccionan con una superficie de uno en uno, de manera secuencial. Es muy parecido al método de CVD, pero en este caso, la reacción se divide en dos "medias" reacciones, manteniendo los materiales precursores separados durante la reacción.

Otro método es el depósito por baño químico (CBD del inglés chemical bath deposition). Éste consiste en la precipitación controlada del material (por ejemplo, CdS) que se desea obtener sobre un sustrato caliente mediante un sistema de reacciones químicas que tienen lugar en disoluciones acuosas, de forma tal que la precipitación ocurre uniformemente sobre el sustrato.

También se usa el método de sputtering, el cual consiste en la extracción de átomos de la superficie de un electrodo debido al intercambio de momento con iones que 
bombardean los átomos de la superficie de un material. Como en el proceso de sputtering se produce vapor del material del electrodo, es también un método utilizado en la deposición de películas.

Usando algunas de estas técnicas también es posible crecer sistemas de multicapas o heteroestructuras metálicas o semiconductoras. Normalmente, las capas pueden tener espesores que varían desde unos nanómetros hasta algunas micras. Estos sistemas son muy importantes en la fabricación de dispositivos electrónicos que se usan en aparatos tales como lectores de discos compactos, teléfonos celulares, diodos emisores de luz o fotoceldas solares.

Las películas delgadas confinan la radiación electromagnética a la propagación libre en sólo dos dimensiones. Similarmente los pozos cuánticos confinan a los electrones a moverse libremente en dos dimensiones. Esto significa que reducir el tamaño de un sistema produce el confinamiento de las partículas.

Si en lugar de fabricar una película delgada fabricamos un alambre, entonces el confinamiento es bidimensional, el movimiento libre se reduce exclusivamente a una dimensión. Para la propagación de la radiación electromagnética estos sistemas se llaman fibras ópticas, mientras que para los electrones se llaman alambres cuánticos. Los alambres se pueden fabricar con técnicas litográficas y por métodos químicos y electroquímicos.

Finalmente, si hacemos que el sistema ahora tenga las tres dimensiones finitas, entonces cualquier partícula estará confinada en las tres direcciones. Este tipo de estructuras son sistemas que se fabrican para inducir el confinamiento tridimensional. Cabe mencionar que las estructuras que confinan a las partículas en tres dimensiones y cuyo tamaño está en los nanómetros también se les llama puntos cuánticos. Una de las técnicas de crecimiento de los puntos cuánticos de semiconductores es el método de autoensamble. En el proceso se deposita un semiconductor sobre un sustrato. La diferencia en el parámetro de red entre el sustrato y el material que se deposita debe ser igual o mayor al 6\%, lo que induce un desacople entre los materiales permitiendo que el material depositado forme islas de diferentes figuras geométricas y tamaños.

En las nanopartículas (objetos de tamaños entre 1-100 nm) el confinamiento también es en tres dimensiones. Es por esto que tienen también propiedades distintas a las de partículas de mayor tamaño y se piensa que pueden ser usadas en muchas aplicaciones en la industria, como la optoelectrónica y la medicina. Las nanopartículas se fabrican en forma económica usando métodos químicos y biológicos. Entre los primeros tenemos, por ejemplo, el modo sol-gel, el de micro-emulsiones. El método sol-gel se inicia con la síntesis de una suspensión coloidal de partículas sólidas o cúmulos en un líquido (sol) y la hidrólisis y condensación de éste sol para formar un material sólido lleno de solvente (gel). El solvente se le extrae al gel simplemente dejándolo reposar a temperatura ambiente durante un periodo de tiempo llamado envejecimiento, en el cual el gel se encogerá expulsando el solvente y agua residual. Al finalizar el tiempo de envejecimiento, el material se somete a un tratamiento térmico para eliminar los solventes y agua que permanecen en el material. Las microemulsiones son mezclas líquidas estables de aceite, agua y un surfactante. A escalas macroscópicas, las micoremulsiones son sistemas homogéneos, pero son heterogéneos a escala molecular ya que existen fases de agua y aceite separados por el surfactante. El método de microemulsiones consiste en confinar los precursores de las nanopartículas en uno de los dominios del sistema, con el fin de facilitar la reacción y controlar el tamaño de la nanopartícula. En el método de aspersión pirolítica (spray pirolisis) se usa un sus- 
trato (metal, vidrio, etc.) colocado dentro de un calefactor al cual se le puede graduar la temperatura y se le hace incidir un aerosol formado por un gas portador (inerte al sistema) más una solución que contiene una sustancia, la cual, al descomponerse sobre la superficie del sustrato, producirá la naonopartícula.

Nanocompositos. Compositos son materiales compuestos por dos o más ingredientes en los cuales al que se encuentra en mayor porcentaje se le denomina matriz y al de menor porcentaje carga. En el caso de los nanocompositos, la carga debe de tener tamaños nanométricos.

Es evidente que es muy importante la fabricación de nanomateriales, por lo que en México se han creado centros de investigación con enfoque principal en estudiar la preparación, realizar la caracterización y buscar las aplicaciones en la industria. En este capítulo presentaremos un reporte de cómo se está desarrollando la investigación en nanomateriales en México. Para ello consultamos las páginas electrónicas de las instituciones y usamos los reportes que se han hecho en las reuniones de especialistas sobre el tema, que han sido organizadas por el Consejo Nacional de Ciencia y Tecnología (Conacyt) de México y otras instituciones académicas. Hemos tratado de incluir el mayor número de instituciones posible, pero en este capítulo no incluimos las nanoestructuras de carbono que serán discutidas en el siguiente. Aplicaciones específicas se presentarán también posteriormente.

\section{Universidad Nacional Autónoma de México [1]}

\section{A. Centro de Nanociencias y Nanotecnología (CNyN) (Ensenada, Baja California) [2]}

El CNyN tiene como misión desarrollar investigación científica del más alto nivel tanto teórica como experimental, básica y orientada a la aplicación tecnológica, en temas de frontera en el campo de los nanomateriales. En la actualidad, cuenta con 5 departamentos: Nanoestructuras, Física teórica, Fisicoquímica de materiales, Nanocatálisis y Materiales Avanzados. Cada departamento tiene sus propias líneas de investigación.

En el Departamento de Nanoestructuras las líneas de investigación son: 1) síntesis de nanomateriales; 2) nanotubos de carbono; 3) metales, sulfuros y óxidos con propiedades electrocatalíticas; 4) semiconductores luminiscentes nanoestructurados; 5) cálculos de primeros principios de la estructura electrónica de materiales y, 6) determinación de la nanoestructura cristalina y electrónica de superficies sólidas y materiales nanoestructurados.

El Departamento de Física Teórica tiene como líneas de investigación: 1) nanomagnetismo y espintrónica; 2) propiedades electromagnéticas de superficies y sistemas inhomogéneos; física estadística de sistemas desordenados y fuera de equilibrio y, 3) cálculos de propiedades estructurales y electrónicas de materiales

El Departamento de Fisicoquímica de nanomateriales desarrolla las siguientes líneas de Investigación: 1) propiedades mecánicas de películas delgadas; 2) producción y caracterización de nanopartículas metálicas; 3) propiedades químicas y físicas de los nitruros de metales de transición; propiedades estructurales, eléctricas y magnéticas de sistemas cristalinos; 4) obtención de las propiedades ópticas de películas delgadas de mediante espectroscopias electrónicas; 5) propiedades fisicoquímicas de nanopartículas y cúmulos de plata y oro; materiales luminiscentes; y 6) estudio de nanoestructuras utilizando sistemas biológicos como mediador. 
El Departamento de Nanocatálisis desarrolla las líneas de investigación que a continuación se detallan: 1) estudio de sulfuros de metales de transición nanométricos soportados y no soportados para reacciones de hidroprocesamiento; 2) estudio de nanopartículas de metales preciosos (Pt, $\mathrm{Pd}, \mathrm{Ru}, \mathrm{Rh}, \mathrm{Au}$, etc.) sobre soporte nanoestructurados y, 3) uso de las zeolitas para sus aplicaciones catalíticas.

Por último, el Departamento de Materiales Avanzados se dedica al desarrollo de las líneas de investigación en: 1) materiales multiferroicos y ferroeléctricos; 2) interacción luz-materia y, 3) nanopartículas semiconductoras en matrices zeolíticas.

\section{B. Instituto de Física (IF) (Ciudad Universitaria) [3]}

El Instituto de Física (IF) de la UNAM está formado por seis departamentos: Estado Sólido; Física Experimental; FísicaQuímica; Física Teórica; Materia Condensada y, Sistemas Complejos. En todos los departamentos se realzan investigaciones relacionadas con los nanomateriales. De alrededor de 200 artículos publicados cada año en revistas indizadas por personal de instituto, cerca de 40 son en nanociencia. El Instituto de Física de la UNAM fundó la red de grupos de investigación en nanociencias (REGINA).

El Instituto cuenta con laboratorios para la fabricación y estudio de nanomateriales. Algunos de los proyectos de investigación que se están realizando en el IF sobre el tema son: 1) transporte de carga y generación de segundo armónico en nanomateriales producidos por el método sol-gel; 2) propiedades ópticas de nanopartículas; 3) filtros y dispositivos solares a partir de óxidos conductores transparentes nanoestructurados; 4) deformación controlada de nanopartículas metálicas embebidas en matrices de sílice usando la irradiación iónica; 5) información cuántica a partir del control de la morfología y de las propiedades óptica lineales y no lineales de nanocompositos metálicos; 6) reconocimiento molecular utilizando nanopartículas quirales; caracterización y escalamiento en la producción de nanopartículas de MxOy (Fe, Cr, Co y Gd) sintetizadas por el método de biosíntesis; 7) caracterización y evaluación preclínica de un nano-sistema dirigido de quimio-radiación para el tratamiento del cáncer cérvico uterino; 8) estructura y reactividad de nanopartículas de Au-Ag soportadas; 9) estudio de la estructura cristalina de materiales a nanoescala a base de metales de transición con propiedades magnéticas, ópticas y catalíticas; 10 ) deformación controlada de nanopartículas metálicas en sílice mediante la irradiación iónica; 11) formación de nanopartículas metálicas en sistemas de películas delgadas de $\mathrm{SiO}_{2} /$ metal y $\mathrm{Si}_{3} \mathrm{~N}_{4} /$ metal; 12 ) fotoconductividad y luminiscencia en materiales amor-

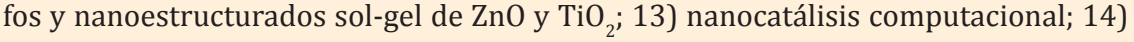
propiedades ópticas de nanoestructuras quirales; 15) producción de nanocristales metálicos anisotrópicos en sílica; 16) estudio de sistemas nanométricos incluidos en materia suave para aplicaciones en nanobiotecnología y nanotecnología utilizando técnicas de corte en un ultramicrotomo criogénico de última generación; 17) síntesis y caracterización de nanopartículas de metales de transición por biorreducción; 18) cambios en las propiedades ópticas de películas de $\mathrm{ZnO}_{2}$ por la irradiación con iones; 19) incorporación de nanocúmulos metálicos y efectos de la radiación.

\section{Instituto de Biotecnología (Cuernavaca, Morelos) [4]}

El Instituto de Biotecnología es una entidad universitaria localizada en Cuernavaca, Mor., y realiza investigación de excelencia académica para el desarrollo de la biotecno- 
logía moderna, generando conocimiento en diversas áreas, incluyendo las nanociencias. Por ejemplo, tratan sistemas relacionados con la nanobiocatálisis; y ha creado recientemente una línea de investigación sobre nanobiotecnología. Se estudian diferentes sistemas nanoestructurados entre los que se pueden mencionar: los materiales nanoporosos, las nanofibras, los nanotubos y las nanopartículas. Algunas de las ventajas que la nanobiocatálisis ofrece se relacionan con la alta superficie específica y la alta carga enzimática, mayor termoestabilidad y mayor estabilidad operacional. En el estudio de nanopartículas se incluyen las partículas magnéticas.

\section{Instituto de Ciencias Físicas (Cuernavaca, Morelos) [5]}

El Instituto de Ciencias Físicas de la UNAM tiene en sus grupos de investigación interés en las nanociencias.

El Grupo de Ciencia de Materiales tiene actualmente entre sus proyectos: 1) la fabricación de nanopartículas de $\mathrm{TiO}_{2}$ y $\mathrm{ZnO}$ en zeolitas para aplicaciones catalíticas; 2) estudios de la influencia de la composición y velocidad de solificación sobre las propiedades mecánicas y electroquímicas de aleaciones nanoestructuradas con base en aluminio y, 3) la síntesis y caracterización de aleaciones metálicas nanoesrtructuradas a base de aluminio.

El Grupo de Física Teórica y Computacional desarrolla proyectos en nanociencias sobre: 1) dispersiones coloidales y su agregación; 2) microestructura y reología de cristales líquidos poliméricos; 3 ) memoria termoelástica anómala en cristales líquidos poliméricos y, 4) epi y nano óptica lineal y no lineal.

En el Laboratorio de Nanopolimeros y Coloides se trabaja en varias líneas de investigación que involucra la nanociencia. Podemos nombrar los siguientes: 1) estudios de nanocompuestos poliméricos, polímeros y nanocompuestos inteligentes; 2) estudios de microestructura por sincrotrón, viscoelasticidad y microestructura. Dentro de las líneas de investigación se desarrollan los siguientes proyectos: microestructura y reología de cristales líquidos poliméricos; correlación nanoestructura y propiedades físicas en nanocompuestos poliméricos; memoria termoelástica anómala en cristales líquidos poliméricos elastoméricos; poliméricos entrecruzados, nanoindentación y microestructura en compuestos a base de poliacrílicos y Policloruro de Vinilo (PVC del inglés polyvinyl chloride) reforzados con nanoarcillas.

\section{E. Instituto de Investigaciones en Materiales (Ciudad Universitaria) [6]}

La investigación de nanosistemas es uno de los temas de investigación en el Instituto de Investigaciones en Materiales de la UNAM. En el Departamento de Materiales Metálicos y Cerámicos los objetivos principales son investigar las propiedades físicas, químicas, mecánicas y microestructurales de materiales cerámicos y metálicos a través de sus diferentes líneas de investigación, desarrollar nuevas tecnologías y desarrollar nuevos materiales metálicos y cerámicos. En el Departamento de Materia Condensada y Criogenia se estudian las propiedades de transporte electrónico, magnéticas y termodinámicas de materiales a temperaturas bajas. Un enfoque es en sistemas electrónicos altamente correlacionados superconductores, sistemas de baja dimensionalidad (como nanotubos y nanoalambres), óxidos complejos entre otros. Se estudia la síntesis de nuevos materiales en diversas formas: películas delgadas, materiales nanoestructurados y en volumen. A los sistemas les estudian sus características 
estructurales, propiedades electrónicas, térmicas, ópticas, mecánicas y magnéticas, y las posibles aplicaciones tecnológicas. En la parte teórica se realizan cálculos de las propiedades físicas y químicas de nuevos materiales. El Departamento de Reología y Mecánica de Materiales también estudia sistemas nanoestructurados: los nanocompuestos poliméricos. Otra línea de investigación en nanociencia es la caracterización fisicoquímica y uso en matrices poliméricas de retardantes de flama (nano- y micrométricos de silicio, fósforo y aluminio). Específicamente, se realizan estudios de nanomateriales y nanoestructuras en los siguientes sistemas: 1) síntesis y caracterización de nanopartículas metálicas y cerámicas; 2) síntesis y caracterización de nanoestructuras de óxido de zinc y óxido de fierro; 3) estudios de las propiedades de transporte en nanotubos de carbón sometidos a altas presiones; 4) depósito y caracterización de nanoestructras de carbón por métodos asistidos por plasmas; 5) síntesis y caracterización de películas delgadas de materiales cerámicos nanoestructurados para celdas de combustible de óxidos sólidos de temperatura intermedia; 6) estudios de fenómenos de absorción y transporte en materiales nanoporosos diseñados con funcionalidad específica; 7) fabricación de nanocompuestos de matriz elastomérica para desarrollo de materiales impermeables a sustancias químicas; 8) estudios de las propiedades electrónicas y estructurales de nanocúmulos metálicos; 9) estudios de las propiedades magnéticas en cúmulos nanométricos de metales de transición; 10) investigación teórica mediante simulación numérica del autoensamblado de sistemas de nanopartículas; 11) investigación de propiedades magnéticas de nanopartículas magnéticas; 12) estudios básicos y aplicaciones de películas delgadas de compuestos aislantes de silicio depositadas por plasma; 13) depósito de películas delgadas de silicio y compuestos de silicio nanoestructurados por técnicas asistidas por plasma (PECVD y RPECVD), utilizando halogenuros de silicio; 14) investigación de las propiedades catalíticas de óxiods metálicos nanoestructurados; 15$)$ estudios de la morfología de las superficies de cúmulos de silicio depositados sobre diferentes sustratos a partir de haluros de silicio por PECVD; 16) crecimiento y caracterización de películas delgadas de compuestos de silicio por la técnica de CVD asistida por plasma a partir de la descomposición de diclorosilano y amoniaco; 17) obtención y estudio de la luminiscencia y electroluminiscencia en sistemas de multicapas de compuestos de silicio y en materiales aislantes con nanopartículas de silicio embebidas; 18) obtención de nanoclusters metálicos por sputtering y modificados por tratamientos con plasmas; 19) obtención y caracterización de silicio polimorfo nanoestructurado por la técnica de PECVD para su uso como material en celdas solares; 20) preparación de películas delgadas y nanocompositos de carbono-metal por métodos asistidos por plasmas y, 21) preparación de materiales diversos en películas delgadas mediante la combinación de procesos sol-gel y espin coating.

\section{F. Centro de Investigaciones en Energía (CIE) (Temixco, Morelos) [7]}

El CIE desarrolla investigación sobre energía desde las áreas físico matemáticas, químico biológicas, económicas y de las ingenierías, así como de nuevas tecnologías para la generación, transformación y uso de energía, para contribuir al desarrollo sustentable del país. El CIE está dividido en tres departamentos: Materiales Solares, Sistemas Energéticos y Termociencias.

El Departamento de Materiales Solares estudia sistemas fotovoltaicos, dispositivos ópticos y optoelectrónicos, y la evaluación de sistemas fotovoltaicos. El Departamento 
está formado por tres coordinaciones: a) Recubrimientos Ópticos y Optoelectrónicos; b) Solar-Hidrógeno: Celdas de Combustión y, c) Superficies, Interfaces y -Materiales Compuestos.

a) La Coordinación de Recubrimientos Ópticos y Optoelectrónicos tiene como objetivos la investigación básica y aplicada de recubrimientos aislantes, conductores y semiconductores fabricados por el depósito químico en solución, en vapor fisicoquímico, y técnicas relacionadas y derivadas de éstas. Desarrollo de dispositivos ópticos y optoelectrónicos útiles para el aprovechamiento de la energía solar, para uso en fuentes no-convencionales de energía y para preservación de fuentes convencionales de energía. Las líneas de investigación que se desarrollan son: preparación de películas delgadas de semiconductores por procesos químicos y fisicoquímicos; desarrollo de recubrimientos controladores solares de semiconductores laminados en vidrio; desarrollo de materiales para dispositivos ópticos y optoelectrónicos; desarrollo de fotodetectores, celdas solares, generadores termoeléctricos, controladores de radiación solar, sensores de gases; caracterización optoelectrónica de materiales y dispositivos; desarrollo de materiales mediante multicapas de semiconductores.

b) La Coordinación de Solar-Hidrógeno: Celdas de Combustión tiene como objetivos principales la síntesis y caracterización de materiales para su aplicación en celdas solares, la preparación de celdas de combustible, producción y almacenamiento de hidrógeno, baterías de hidruro metálico y litio, y supercapacitores, la fabricación y caracterización de bioenergía y biocombustibles, la instalación y caracterización de sistemas híbridos de fotovoltaica-hidrógeno-celda de combustible. Las líneas de investigación son: celdas solares de películas delgadas y nanoestructuradas basadas en $\mathrm{CuInSe}_{2}$, $\mathrm{CdTe}$ y $\mathrm{TiO}_{2}$; celdas de combustible; producción, almacenamiento y aplicación de hidrógeno; nanociencia y nanotecnologia aplicada en la síntesis de materiales, fabricación de dispositivos y generación de energía; baterias de hidruro metálico y litio; supercapacitores; bioenergía y biocombustible; y preparación y caracterización de películas delgadas semiconductoras, para su empleo como dispositivos controladores de la radiación solar en edificaciones

c) La Coordinación de Superficies, Interfaces y Materiales Compuestos tiene como objetivos generar conocimiento en la preparación y caracterización fisicoquímica de los sistemas multifásicos y sus interfaces, análisis de muestras por técnicas espectroscópicas y microscópicas, y de la aplicación de modelos teóricos adecuados. Las líneas de investigación de esta Coordinación son: preparación de materiales compuestos polímero conductor/polímero, polímero conductor/ semiconductor inorgánico, polímero conductor/fulerenos, para aplicaciones múltiples (sensores, diodos rectificadores, recubrimientos protectores, etc.); síntesis de nanopartículas semiconductoras en forma de emulsión y película delgada, para aplicaciones fotocatalíticas y fotoelectrolíticas (producción de hidrógeno); desarrollo de materiales compuestos a partir de fulerenos y otras formas de carbón, para procesos de conversión y almacenamiento de energía; desarrollo de compuestos del tipo II-IV por medio de la técnica de sol-gel para el diseño de conductores transparentes y dispositivos controladores de la radiación solar; fabricación de fotoelectrodos y dispositivos fotoelectroquímicos, fotocatalíticos, y fotoelectrolíticos; deposito químico y electroquímico de pelí- 
culas electrocrómicas inorgánicas y poliméricas, así como el desarrollo de dispositivos electrocrómicos ahorradores de energía (ventanas inteligentes); física de superficies de materiales semiconductores; caracterización de interfaces semiconductor/líquido, semiconductor/sólido, semiconductor/gas, mediante técnicas electroquímicas y fotoelectroquímicas.

En el Departamento de Sistemas Energéticos se realizan estudios sobre sistemas para el aprovechamiento de la energía solar y geotérmica; de planificación energética y sistemas de refrigeración, bombas de calor y transformadores térmicos. Está integrado de las siguientes coordinaciones: a) Concentración Solar; b) Geoenergía; c) Planeación Energética y, d) Refrigeración y Bombas de Calor.

Departamento de Termociencias, aquí se llevan a cabo estudios básicos y aplicados sobre fenómenos de termodinámica de procesos irreversibles, mecánica estadística y física del estado sólido; además, se estudia la transferencia de energía y masa en varios sistemas: flujo en canales, convección natural, flujos oscilatorios, entre otros. Está formado de dos coordinaciones: a) Física Teórica y b) Transferencia de Energía y Masa. En la primera se investiga la propagación de radiación electromagnética en cristales fotónicos; los estudios tratan con las propiedades ópticas de nanoestructuras de silicio poroso y los efectos de desorden en los sistemas, enfatizando los aspectos teóricos y experimentales. Las estructuras que se tratan se forman de capas con diferentes porosidades que pueden ser útiles en aplicaciones ópticas como filtros, espejos y microcavidades.

\section{G. Instituto de Química (Ciudad Universitaria) [8]}

Está formado por los Departamentos de: Bioquímica; Fisicoquímica; Productos Naturales; Química Inorgánica y, Síntesis Orgánica. En el Departamento de Síntesis Orgánica es donde se preparan y caracterizan sistemas nanoestructurados como son las nanaopartículas. Además, se investigan las interacciones intermoleculares de fulerenos $\mathrm{C}_{60} \mathrm{y}$ $\mathrm{C}_{70}$ con macromoléculas y las reacciones de cicloadición de fulerenos $\mathrm{C}_{60} \mathrm{y} \mathrm{C}_{70}$.

\section{H. Centro de Centro de Ciencias Aplicadas y Desarrollo Tecnológico (CCADET) (Ciudad Universitaria) [9]}

En el CCADET se busca: 1) preparar materiales nanoestructurados con tamaño y forma controlada, así como el desarrollo de metodologías de nanoensamblado; 2) desarrollar herramientas para nanomanipulación y adquisición de señales mediante sondas con resolución nanométrica; 3) desarrollar dispositivos basados en nanoestructuras (sensores, biosensores, purificadores ambientales, lab-on a chip, etc.); 4) desarrollar aplicaciones en áreas estratégicas (medio ambiente, salud, energía, alimentos, entre otras); 5) transferir las tecnologías desarrolladas. Algunos de los proyectos que se desarrollan son: 1) materiales multifuncionales nanoestructurados basados en óxidos mixtos; 2) mecanosíntesis de ferritas nanoestructuradas; mecanosíntesis de óxidos multiferróicos nanoestructurados; 3 ) síntesis de catalizadores heterogéneos basados en oro; 4) efecto del tamaño de cristal del soporte sobre la actividad catalítica CO + 1/2 $\mathrm{O}_{2} \rightarrow \mathrm{CO}_{2} ; 5$ ) reacción de desplazamiento del gas de agua (WGS del inglés Water Gas Shift reaction) $\mathrm{CO}+\mathrm{H}_{2} \mathrm{O} \rightarrow \mathrm{CO}_{2}+\mathrm{H}_{2}$; 6) nanocompositos dendrímero-nanopartículas de metales-nobles y sus potenciales aplicaciones medioambientales y farmacológicas; 7) generación de los catalizadores dendriméricos; 8) estudio de la dependencia 
de la rugosidad del sustrato con la anisotropía magnética en películas de permaloy; 9) análisis topográfico para diferentes sustratos; 10) películas delgadas de oro nanoestructuradas usando como molde la capa barrera de alúminas anódicas porosas; 11) desarrollo de sistemas metálicos nanoestructurados para ser usados en espectroscopía vibracional; 12) adsorción de contaminantes orgánicos de aguas residuales por silicalita-1 derivada de la cáscara de arroz; 13) nanoestructuras bioinorgánicas; 14) síntesis de nanopartículas de $\mathrm{TiO}_{2}$ a partir de alcóxidos de titanio.

\section{Centro de Física Aplicada y Tecnología Avanzada (CFATA) (Querétaro) [10]}

Algunos de los proyectos del Centro CFATA incluyen el desarrollo de polímeros conductores nanoestructurados por métodos químicos oxidativos tiene como objetivo sintetizar químicamente polímeros conductores nanoestructurados para el desarrollo de materiales compuestos plásticos para aplicaciones antiestáticas. Otro proyecto es del desarrollo de dispositivos piezoeléctricos para aplicaciones en odontología está basado en el desarrollo de sensores piezoeléctricos con la finalidad de ajustar la presión.

En el CFATA se desarrolla la síntesis de películas de diamante usando la técnica del depósito químico en fase vapor (CVD) a partir de la inyección pulsada de precursores líquidos orgánicos. Estos precursores incluyen acetona, etanol y metanol. Síntesis de películas de materiales cerámicos a partir de precursores organometálicos usando un sistema de inyección pulsada por depósito químico en fase vapor (MOCVD). Por otra parte, la caracterización incluye las espectroscópicas la raman e infrarroja, así como la microscopía electrónica de barrido (SEM), mismas que se realizan con equipos disponibles en otros laboratorios de este centro. La aplicación de películas de diamante es otra de las líneas de investigación en desarrollo. Dada las excepcionales características del diamante, se intenta desarrollar novedosas aplicaciones dentro de las que se encuentran los detectores de radiación y las herramientas de corte, entre otras.

Hay líneas de investigación que se relacionan con el modelado teórico de las estructuras y propiedades de transporte de materiales no periódicos, como los cuasicristales. Se desarrollan métodos matemáticos para la cristalografía moderna y formación de patrones en sistemas biológicos. Hay interés en los estudios de la propagación de ondas en medios nanoestructurados, en particular en la propagación de ondas de ultrasonido en cristales y cuasicristales fonónicos y ondas hidriodinámicas en cristales y cuasicristales hidrodinámicos. Los cuasicristales que se estudian son de aleaciones metálicas con orden de translación de largo alcance pero con una simetría puntual incompatible con la periodicidad (ejes de rotación de orden $5,8,10,12$ ). Los estudios se centran en la estructura atómica y las propiedades de transporte de estos materiales y la geometría de adoquinados no periódicos.

Se preparan los polímeros conductores nanoestructurados por métodos químicos oxidativos. El objetivo es sintetizar químicamente polímeros conductores nanoestructurados para el desarrollo de compuestos plásticos para aplicaciones antiestáticas. La investigación de nuevos materiales toma en cuenta también el desarrollo de la óptica teórica, además de emplear la microscopía electrónica. Se estudia la termodinámica de sistemas pequeños y se desarrollan de sistemas nanométricos. Se desarrollan nuevos materiales: polímeros, cerámicos, metales e híbridos. En el CFATA se investigan biomateriales cerámicos y microestructuras mediante difracción de rayos-X por el método de polvos. Se desarrollan materiales nanoestructurados con propiedades catalíticas. Además, se preparan materiales mesoporosos, nanoestructurados y nanopartículas. 


\section{J. Facultad de Ciencias (Ciudad Universitaria) [11]}

La Facultad de Ciencias de la UNAM realiza investigación en nanociencias a través de los posgrados en ciencia en ingeniería de materiales y en ciencias físicas. Algunas de las líneas de investigación que se desarrollan son: propiedades magnéticas de materiales nanoestructurados y el estudio de nanopartículas metálicas y bimetálicas en óxidos metálicos.

\section{K. Facultad de Química (Ciudad Universitaria) [12]}

En la Facultad de Química se sintetizan nanopartículas en forma coloidal. ${ }^{1}$ Este método de preparación no requiere de instrumentación costosa y complicada. Además, las dispersiones coloidales diluidas permiten controlar las dimensiones de las nanopartículas. Algunos materiales nanoestructurados que se fabrican en la facultad son: partículas metálicas de $\mathrm{Ag}$, $\mathrm{Bi}$, $\mathrm{Fe}, \mathrm{Cu}$, $\mathrm{Ni}$ y Co; óxidos metálicos: $\mathrm{ZnO}, \mathrm{Cu}_{2} \mathrm{O}, \mathrm{Bi}_{2} \mathrm{O}_{3}, \mathrm{SnO}_{2}$, $\mathrm{Fe}_{3} \mathrm{O}_{4}$ y $\mathrm{TiO}_{2}$; compositos: Ag@CNT’s, Ag@Cgrafito, Ag@MoS $, \mathrm{Ag} @ \beta-\mathrm{CD}, \mathrm{Bi}_{2} \mathrm{~S}_{3} @ \mathrm{Cgra}-$ fito, $\mathrm{Bi}_{2} \mathrm{~S}_{3} @ \mathrm{CNT}$ 's, Ag-ZnO, ZnO@arcillas multilamelares, $\mathrm{SiO}_{2} @ \mathrm{ZnO}$; y sulfuros metálicos: $\mathrm{RuS}_{2}, \mathrm{CdS}, \mathrm{MoS}_{4} @ \mathrm{CdS}, \mathrm{SrS}, \mathrm{M}(\mathrm{II}) @ \mathrm{CdS}$, dendrímeros Gn-M(II)@CdS.

Perspectivas y aplicaciones: 1) degradación de gases contaminantes a partir de fuentes de emisión fijas, tales como chimeneas de industrias diversas y ladrilleras, asistida con compositos hechos a base de materiales inorgánicos nanoestructurados; 2) aniquilación de compuestos orgánicos persistentes (bifenilos policlorados, pesticidas organofosfatados y compuestos orgánicos volátiles), asistida con nanopartículas de metales cerovalentes; 3) obtención de materiales nanoestructurados hechos a base de bismuto, que funcionen como agentes contrastantes, no tóxicos, para imágenes de resonancia magnética nuclear (NMR del inglés nuclear magnetic resonance) y tomografía por emisión de positrones (PET del inglés positron emission tomography); 4) preparación de membranas recubiertas con nanopartículas ZVM (metales de valencia cero) para el tratamiento de aguas residuales industriales; 5) estudio fisicoquímico de la interacción de DNA y nanopartículas inorgánicas; 6) producción masiva de nanopartículas inorgánicas con dimensiones inferiores a $10 \mathrm{~nm}$, usando procedimientos químicos compatibles con el medio ambiente y condiciones de reacción normales; 7) obtención de compositos hechos a base de grafito y nanoestructuras inorgánicas con aplicaciones en la electrónica y la industria aeronáutica.

\section{Instituto Politécnico Nacional [13]}

\section{A. Centro de Nanociencia y Micro-Nanotecnología [14]}

Laboratorios propuestos: 1) caracterización, con equipos de difracción de rayos X, espectroscopías, microscopías y nanomanipulación, propiedades fisicoquímicas; 2) dispositivos y microelectrónica; 3) síntesis y procesamiento.

Tiene como líneas de investigación: 1) energía; 2) medio ambiente; 3) biotecnología; 4) microelectrónica y MEMS; 5) materiales y biomateriales.

${ }^{1}$ Díaz, D. <http://www.nanored.org.mx/documentos/David\%20Diaz.pdf>. 


\section{B. Escuela Superior de Física y Matemáticas del IPN [15]}

En esta sección describiremos las nanoestructuras cuánticas que se construyen de semiconductores tanto simples como compuestos. Las estructuras se fabrican con capas delgadas de diferentes materiales. Por ejemplo, en la Escuela Superior de Física y Matemáticas (ESFM) del Instituto Politécnico Nacional hay un grupo de investigadores que se dedican a la fabricación y caracterización de sistemas multicapas semiconductoras. Entre los materiales que se estudian están: CdS, CdTe, Si, compósitos semiconductores y superconductores y celdas solares orgánicas. Se investigan heteroestructuras formadas por estos materiales para su uso como celdas solares. En la actualidad se fabrican también celdas solares orgánicas.

Las técnicas de crecimiento con las que cuenta la ESFM del IPN incluyen el sputtering, complementado con el devastador iónico y el electro-adelgazador de muestras. También trabajan con el método depósito por baño químico (CBD). En la fabricación de películas de CdS se depositaron en vidrio conductor $\mathrm{SnO}_{2}: \mathrm{F}$ en un baño de reactivos químicos en concentraciones determinadas, temperaturas y tiempos de depósito, conteniendo: cloruro de cadmio, cloruro de amonio, hidróxido de amonio y tiourea. Por otro lado con el método de depósito por aerosol se pueden fabricar compósitos semiconductores y superconductores. Un compósito es cualquier material constituido por más de un componente, un ejemplo es el material superconductor $\mathrm{Bi}-\mathrm{Pb}-\mathrm{Sb}$-Sr$\mathrm{Ca}-\mathrm{Cu}-\mathrm{O} / \mathrm{CdS}$ el cual se ha preparado y caracterizado en la ESFM.

Las caracterizaciones de los sistemas se llevan a cabo con diferentes técnicas. Las propiedades eléctricas se estudian con mediciones de cátodo-luminiscencia. La morfología se investiga usando la microscopia electrónica. La espectroscopia Raman y la foto-luminiscencia se emplean para estudiar las propiedades ópticas.

\section{Centro de Investigación y de Estudios Avanzados del IPN [16]}

\section{A. Departamento de Física (Zacatenco) [17]}

Cuenta con la Sección de Física del Estado Sólido formado por dos grupos: la Sección de Física del Estado Sólido (SFES) y el Grupo de Nanoestructuras Semiconductoras [18]; se dedican a la preparación y caracterización de sistemas que se forman de capas semiconductoras simples y compuestos. SFES cuenta con diferentes técnicas para el crecimiento de películas delgadas las cuales son: erosión catódica por radio frecuencia, baño químico, sublimación en espacio cerrado, rocío pirolítico, depósito en fase vapor asistido por plasma, epitaxia por haces moleculares, epitaxia en fase líquida, screen printing, sol-gel y ablación láser. Por otro lado, cuentan con equipos para la caracterización eléctrica, óptica, estructural y química de los materiales.

La caracterización eléctrica se realiza con la espectroscopía transitoria de niveles profundos, fotoconductividad, medición de capacitancia contra voltaje a alta y baja frecuencia y de corriente contra voltaje. Las propiedades ópticas se investigan con fotoluminiscencia, espectroscopía en el ultravioleta, visible e infrarrojo en sus modos de absorción y transmisión, espectroscopía fotoacústica, elipsometría espectral, espectroscopía Raman, fotorreflectancia y reflectividad. Para determinar las estructuras de las muestras se emplean los microscopios electrónicos de barrido y transmisión, de fuerza atómica y de tunelamiento. Las propiedades químicas de los materiales 
se caracterizan con la espectroscopía de rayos x de energía dispersa, espectroscopía electrónica Auger y fotoemisión. Asimismo, se trabaja con técnicas fototérmicas para realizar la caracterización de las propiedades térmicas. Además, se cuenta con un difractómetro de rayos x con aditamentos para realizar mediciones a ángulos rasantes y a altas temperaturas necesarios para el análisis de películas delgadas.

También se han realizado estudios en películas nanométricas luminiscentes, dieléctricas y superconductoras depositadas por rocío pirolítico. Las propiedades luminiscentes de las películas se consiguen al ser impurificadas con diversos materiales a fin de generar emisiones luminosas policromáticas en el rango de la luz visible. Estos sistemas luminiscentes tienen aplicaciones prácticas en el desarrollo de las pantallas. La técnica es particularmente adecuada para el depósito sobre áreas grandes a un costo muy bajo. Este grupo ha sido pionero en el uso de la técnica para la obtención de películas luminiscentes, dieléctricas y superconductoras.

El Laboratorio de Nanoestructuras Semiconductoras (NanoSem) [19] cuenta con un grupo de profesores, estudiantes y auxiliares de investigación dedicados al crecimiento y caracterización de superficies, películas delgadas y heteroestructuras semiconductoras de baja dimensionalidad para aplicación en dispositivos optoelectrónicos, particularmente diódos luminiscentes, láseres semiconductores, fotodetectores, celdas solares, etc. Las estructuras se preparan mediante técnicas como la epitaxia de haces moleculares (MBE, molecular beam epitaxy), epitaxia de capas atómicas (ALE, atomic layer epitaxy), epitaxia de haces pulsados de submonocapas (SPBE, submonolayer pulsed beam epitaxy). Los estudios se complementan con la caracterización óptica, electrónica, química, estructural y eléctrica de semiconductores y sus nanoestructuras semiconductoras.

Las heteroestructuras que se construyen de CdSe son de capas delgadas con espesores pequeños de alrededor de tres monocapas atómicas. Esta capa puede usarse como un pozo cuántico donde las energías de los electrones se cuantizan. Se ha detectado experimentalmente la emisión luminiscente verde a temperatura ambiente del pozo cuántico subnanométrico.

\section{Departamento de Física Aplicada (Mérida) [20]}

Está integrada por cinco cuerpos académicos, de los cuales, cuatro tienen temas relacionados con los nanomateriales: física de materiales; fisicoquímica, medio ambiente e infraestructura; nano y biomateriales; y física aplicada. Algunos de los proyectos que están relacionados con la nanociencia son: estudio cuantitativo de celdas solares de CuInGaSe, películas delgadas de óxidos transparentes semiconductores de CdTe tipo-n y/o tipo-p, estructura atómica y electrónica local en pozos cuánticos de semiconductores II - VI, nanoestructuración y propiedades físicas de capas delgadas de aleaciones metálicas, influencia de la composición de la matriz en la resistencia a la corrosión de compósitos $\mathrm{Al}-\mathrm{Si}-\mathrm{Mg} / \mathrm{SiCp}$ obtenidos por infiltración reactiva, y estudio de primeros principios de sólidos y nanoestructuras.

\section{Unidad Querétaro [21]}

La Unidad Querétaro del Cinvestav está formada por cuatro cuerpos académicos: 1) materiales multifuncionales; 2) nanomateriales; 3) materiales para aplicaciones en energía y medio ambiente y, 4) materiales bioorgánicos. 
Las principales líneas de investigación relacionadas con los nanomateriales son las siguientes: 1) procesamiento de materiales nanoestructurados: por deposición física de vapor y rociado térmico, proceso Sol-Gel o Zeolitas; 2) caracterización de superficies a escala nanométrica por: microscopía de fuerza atómica, nanoindentación, caracterización óptica (espectroscopía Raman entre otras); 3) aplicaciones de materiales nanoestructurados: fotocatálisis, catálisis $\left(\mathrm{O}_{2}\right.$ y $\mathrm{H}_{2}$ principalmente); 4) nanociencia computacional: predicción, caracterización y correlación experimental; caracterización de superficies y simulación; caracterización electrónica, vibracional, térmica, elástica y óptica; nanoestructuras magnéticas, metálicas, iónicas y semiconductoras.

\section{E. Departamento de Ingeniería Eléctrica (Zacatenco) [22]}

Para el desarrollo de la investigación el Departamento de Ingeniería Eléctrica del Cinvestav está organizado en secciones académicas, de las cuales la Sección de Eléctronica de Estado Sólido (SEES) [23] desarrolla proyectos en nanociencias y nanotecnología, y donde se realiza caracterización de semiconductores de las siguiente maneras: 1) eléctrica por efecto Hall, I-V (intensidad-voltaje), C-V (capacitancia-voltage), DLTS (espectroscopía transitoria de nivel profundo, del inglés deep level transient spectroscopy); 2) óptica por fotoluminiscencia y transmitancia; 3) reflectancia, catodoluminiscencia y, 4) estructural por SIMS, microscopía electrónica, microscopía de fuerza atómica, difracción de rayos x. Se estudian, además, materiales semiconductores como silicio monocristalino, silicio amorfo, silicio poroso, semiconductores III-V, semiconductores orgánicos y semiconductores transparentes. Se fabrican dispositivos semiconductores como celdas solares, diodos emisores de luz., OLEDs., TFTs, sensores de gases, sensores MOS basados en transistores de compuerta flotante. Se hace diseño de circuitos integrados VLSI: diseño de circuitos integrados analógicos CMOS, desarrollo de prototipos en tecnología de componentes VLSI digitales- FPGA, diseño de sistemas MEMS (micro-electro-mechanical systems), desarrollo de métodos para análisis de datos basados en técnicas neurodifusas.

\section{Benemérita Universidad Autónoma de Puebla (Puebla) [24]}

\section{A. Centro de Investigación en Dispositivos Semiconductores (CIDS) [25]}

El CIDS es un Centro que pertenece al Instituto de Ciencias de la BUAP, en donde hay dos cuerpos académicos: el CA de Materiales y Dispositivos Semiconductores y el CA de Aplicaciones Tecnológicas de Dispositivos Semiconductores.

El CA de Materiales y Dispositivos Semiconductores desarrolla las siguientes líneas de investigación: 1) desarrollo de materiales y estructuras semiconductores y su caracterización; 2) dispositivos electrónicos y optoelectrónicos, diseño y caracterización; 3) estudios de compuestos semiconductores III-V, sus características y las propiedades optoelectrónicas de sus cristales.

El CA de Aplicaciones Tecnológicas de Dispositivos Semiconductores desarrolla las líneas de investigación: 1) diseño y construcción de celdas termoiónicas y termoeléctricas; 2) diseño de sistemas electrónicos de medición y control; 3) síntesis de nuevos materiales solubles de ftalocianinas con aplicaciones potenciales en optoelectrónica; 4) aplicación de las microondas como fuente alternativa de energía para la 
síntesis de compuestos; 5) estudio de efectos transductores para la obtención de microsensores con semiconductores y para materiales no convencionales compatibles con tecnología microelectrónica; 6) diseño, fabricación y caracterización de circuitos integrados, en especial aplicación a MEMS y biosensores.

\section{B. Instituto de Física 'Luis Rivera Terrazas' [26]}

Este Instituto está organizado en seis cuerpos académicos. Cada cuerpo académico (CA) tiene líneas de investigación, a continuación se mencionas las líneas de investigación que están relacionadas con nanomateriales.

CA de Física Aplicada: 1) física de superficies e interfaces; 2) propiedades ópticas y acústicas de sistemas periódicos artificiales; se estudian las propiedades ópticas de los cristales fotónicos desde enfoques tanto teóricos como experimentales; en particular se desarrolla una línea experimental dedicada al crecimiento y caracterización de estructuras unidimensionales basadas en silicio poroso, la cual se apoya en el estudio teórico de las propiedades ópticas de cristales fotónicos y las acústicas de los cristales fonónicos utilizando diversos enfoques; 3) cristales fotónicos y fonónicos; se estudia la propagación de ondas electromagnéticas y elásticas en sistemas periódicos uni, bi y tridimensionales; se desarrollan modelos teóricos que permitan controlar la propagación de dichas ondas con propósito de aplicaciones tecnológicas; también se estudian las propiedades de estos sistemas desde un punto de vista fundamental, en particular se calculan constantes efectivas mediante métodos de homogeneización.

CA de Materiales Avanzados: 1) superconductividad y magnetismo; 2) materiales avanzados. En esta línea se investigan las propiedades ópticas y acústicas de materiales compositos con estructura periódica, cuasiperiódica, y aleatoria; en particular, se estudia la respuesta óptica de metamateriales fotónicos; se desarrollan y aplican modelos teóricos para la descripción de las propiedades acústicas de metamateriales fonónicos; en este tipo de sistemas también se incluyen las nanoestructuras con diferentes tipos de inclusiones: metálicas, semiconductoras, magnéticas y dieléctricas.

CA de Materiales Complejos, Inteligentes y Nanoestructurados: 1) materiales complejos e inteligentes; en esta línea de investigación se desarrollan nuevas técnicas de estudio experimental y teórico sobre materiales complejos como: compositos, geles, emulsiones, etc.; así como de materiales funcionales también llamados materiales inteligentes, por ejemplo, ferroeléctricos, ferroelásticos, relaxores, fluidos magneto y electro-reológicos, etc.; 2) nanopartículas y nanocompositos, desde el punto de vista experimental se desarrolla el crecimiento y caracterización de nanopartículas metálicas y semiconductoras; el estudio de nanocompositos, particularmente los efectos de matrices activas en las propiedades de las nanopartículas y sus posibles aplicaciones; en el aspecto teórico se estudian las manifestaciones de las fluctuaciones y de los efectos de tamaño cuántico propias de estos sistemas para detectar posibles aplicaciones.

CA de Materiales Fotocatalíticos y Fotoconductivos: 1) propiedades morfológicas y químicas de materiales; el objetivo de esta línea de investigación es el estudio de las características morfológicas y de la composición química de materiales mediante las microscopías óptica, electrónica de barrido (SEM) y de fuerza atómica (AFM), las espectroscopías de rayos-x por dispersión en energía (EDS), de electrones Auger (AES) y fotoelectrónica de rayos-x (XPS), para poder elucidar los mecanismos de crecimiento y su influencia en las propiedades fotocatalíticas y fotoconductivas de los 
materiales; 2) propiedades fotocatalíticas, luminiscentes y fotoeléctricas de materiales; el objetivo de esta línea es la síntesis de materiales y su caracterización mediante las técnicas de cromatografía de gases y de líquidos, espectroscopias de infrarrojo por transformada de Fourier (FTIR), ultravioleta y visible (UV-Vis), fotoluminiscencia, fotoconductividad y efecto Hall, para elucidar los mecanismos de absorción y emisión de luz, la respuesta fotoeléctrica y la eficiencia de los materiales en procesos de óxidoreducción de compuestos orgánicos y de generación de energía.

CA de Física Computacional de la Materia Condensada: 1) cálculos ab initio de la estructura electrónica de átomos, moléculas y sólidos; se realizan estudios de la estructura electrónica de átomos, moléculas y sólidos en general, usando el modelo computacional empleando los métodos de la química cuántica y el estado sólido (HF, pos-Hf, DFT), así como la utilización de códigos apropiados para el estudio de sistemas cristalinos. Se hace énfasis en el estudio del proceso de catálisis en superficies semiconductoras y en la obtención de niveles f de lantánidos que entran como impurezas en diversos cristales. Se estudia también la formación de nanoestructuras; 2) métodos variacionales y su aplicación en sistemas confinados. Utilizando el principio variacional se realizan cálculos sobre átomos y moléculas bajo confinamiento, lo que es de gran interés en el estudio de puntos cuánticos; 3) propiedades ópticas; cálculos de la respuesta óptica de sistemas de dimensionalidad reducida; los materiales que estudian se forman de metales, semiconductores o dieléctricos; se usan modelos macroscópicos de la función dieléctrica; se realizan cálculos de la fuerza de Casimir en nanosistemas; en particular, se estudian las geometrías de placas paralelas de metales, dieléctricos y semiconductores; 4) simulación molecular de líquidos

\section{Facultad de Ciencias Físico-Matemáticas [27]}

Dentro de los 11 CA's que tiene la Facultad, los que desarrollan investigación en nanociencias son:

CA de Física de Materiales: 1) interacción de radiación electromagnética en materia condensada: síntesis y caracterización de películas delgadas mediante los procesos sol-gel, baño químico, evaporación por cañón de electrones y evaporación por filamento. Estudio de mecanismos de transporte.

CA de Óptica Cuántica: 1) haces adifraccionales y solitones ópticos: interacciones no lineales en medios cuadráticos, cúbicos, competencia de no linealidades y medios estratificados; fenómenos no lineales ultrarápidos; óptica de campo cercano; 2) sistemsa fotónicos: detectores fotónicos; láseres de pulsos ultracortos; cristales fotorefractivos; sistemas para biofotónica; trampas de nanofotónica; técnica de barrido en Z; 3) optica cuántica: estados cuánticos de la luz; absorción multifotónica del medio cuantizado; operador de densidad y modelo eléctrico dipolar.

CA de Optoelectrónica y Fotónica: 1) óptica no lineal: generación, propagación e interacción de pulsos luminosos ultracortos y su interacción no lineal con medios reales y sus aplicaciones en fotónica; 2) sistemas optoelectrónicos: estudio del procesamiento de información óptica, sistemas de comunicación óptica, dispositivos y materiales optoelectrónicos, láseres semiconductores, comunicaciones inalámbricas y reconocimiento de patrones; su interés radica en el estudio de las condiciones óptimas de un proceso, modelo o función; 3) láseres y fibra óptica: en el marco de estudios fundamentales y aplicaciones en fotónica, de manera experimental, teórica y numérica se investiga la interacción no lineal o dispersa de radiación electromagné- 
tica en guías de onda, en los procesos de generación, propagación y amplificación de señales codificadas y pulsos ópticos; En estos estudios especial atención se presta al desarrollo de nuevos láseres de fibra óptica.

\section{Centro Universitario de Vinculación (CUV) [28]}

La investigación en el CUV tiene como objetivo contribuir a la generación de nuevos conocimientos en el campo de la ciencia de materiales, difundir, divulgar y transferir al medio académico los conocimientos generados, los preexistentes y los que surjan de la actividad mundial en ese campo y contribuir a la formación y al perfeccionamiento de recursos humanos, tanto a nivel de investigadores como profesionales y técnicos en sus campos de actividad y en otros relacionados con éstos.

Para realizar las actividades antes mencionadas el área de investigación del CUV cuenta con los siguientes temas de investigación.

Nanomateriales: 1) síntesis de materiales mesoporosos de silica con nanopartículas de plata; 2) métodos experimentales para la obtención de sistemas estables de nanopartículas de $\mathrm{Au}, \mathrm{Pd}$ y Pt con una distribución de tamaño bastante cerrada.

Materiales cerámicos: 1) materiales cerámicos para dosimetría; recubrimientos cerámicos por depósito químico en fase vapor (CVD); síntesis de nanopartículas cerámicas para aplicación en catálisis.

Materiales híbridos: 1) síntesis y caracterización de recubrimientos híbridos orgánico-inorgánico a base de silica, PMMA y resina epóxica para la protección de sustratos metálicos; 2) síntesis y caracterización de un material híbrido silica-tiol para la remoción de ion cromo en un sistema acuoso; 3) síntesis y caracterización de materiales híbridos a base de $\mathrm{PMMA}, \mathrm{SiO}_{2}$ y nanopartículas de oro.

Biomateriales: 1) síntesis y caracterización de hidroxiapatita por sol-gel, método hidrotermal y precipitación; 2) crecimiento biomimético de hidroxiapatita sobre templetes orgánicos; 3) síntesis de hidroxiapatita nanoestructurada usando micelas: 4) materiales compuestos de ácido polilactico-hidroxiapatita

\section{Universidad Autónoma de Nuevo León (MonterRey) [29]}

\section{A. Centro de Innovación, Investigación y Desarrollo en Ingeniería y Tecnología (CIIDIT) [30]}

El CIIDIT es un Centro multidisciplinario e integrador de la UANL constituido por laboratorios de última generación para distintas disciplinas de la ingeniería y tecnología. El Centro comprende las áreas emergentes estratégicas para el desarrollo industrial, económico y productivo a nivel regional y nacional. Su objetivo es dar un impulso decisivo al fortalecimiento de los cuerpos académicos de la Universidad, a los posgrados, a las redes multidisciplinarias de investigación y promover la internacionalización de la institución mediante la firma de convenios de vinculación con otros centros e instituciones de prestigio mundial. Relevantes a las nanociencias, el CIIDIT desarrolla las áreas de investigación en nanotecnología y materiales avanzados.

Los proyectos de desarrollo en el área de nanotecnología son: 1) interacción de nanopartículas de plata con HIV-1, con el objetivo de la destrucción de células enfermas; 2) nanopartículas paramagnéticas-conductoras tipo core-shell, para purificación de aguas residuales; 3) nanopartículas de magnetita en quitosán, para sistemas 
de entrega localizada de medicamento y para posicionamiento de nanopartículas en una región específica del cuerpo; 4) nanopartículas de aleación titanio-níquel para activadores eléctricos y/o térmicos.

Los proyectos de desarrollo en el área de materiales avanzados incluyen los siguientes: 1) polímeros aldólicos conjugados para aplicaciones en dispositivos fotoelectrónicos; 2) composito híbrido de resina poliéster-montmorillonita; 3) suspensión de nanofibras de carbono con propiedades electroreológicas, para sensores, actuadores, MEMS y NEMS y, 4) polímeros luminescentes con propiedades de autoensamblaje para biosensores.

\section{A. Facultad de Ciencias Físico Matemáticas [31]}

La maestría y el doctorado en ingeniería industrial cuenta con los laboratorios de diseño molecular (LDM) y nanociencias y nanotecnología. El LDM es un laboratorio para el estudio de propiedades físicas de sistemas a nivel atómico y molecular, a través de técnicas de simulaciones y cálculo numérico basados en principios físicos. El propósito del LDM es contribuir, a través de sus investigaciones y actividades, en la conexión entre los enfoques teóricos y experimentales para el estudio de estructura y propiedades de sistemas de escalas nanoscópicas y microscópicas. Gracias a los vertiginosos avances en las técnicas computacionales para el cálculo de propiedades, simulación, y visualización de sistemas moleculares, esta área ha venido incrementando su importancia en medicina, industria, desarrollo tecnológico, y ciencia básica. Las actividades de investigación que se desarrollan en el LDM incluyen: 1) estudio de propiedades estructurales y termodinámicas de nanoestructuras; 2) desarrollo de modelos para sistemas coloidales y poliméricos; 3) obtención de diagramas de fases termodinámicas y propiedades estructurales de sistemas coloidales; 4) desarrollo de nuevos algoritmos de dinámica molecular para ensambles estadísticos especiales; 5) análisis y simulación de imágenes de microscopía electrónica; 6) diseño de potenciales de interacción a escala atómica.

El Laboratorio de Nanociencias y Nanotecnologías está dedicado a la síntesis, caracterización, y usos de materiales nanoestructurados semiconductores y metálicos. Allí se realizan investigaciones dirigidas a la producción y análisis de nanoestructuras, cuyas características físicas, químicas y mecánicas puedan ser aprovechadas en muy diversas áreas. Cuenta con equipo especializado para producir y caracterizar este tipo de sistemas, y colabora a través de proyectos interdisciplinarios con grupos de investigación nacionales y extranjeros. En conjunto con el Laboratorio de Diseño Molecular de la FCFM, se realizan simulaciones numéricas por computadora que permiten predecir las propiedades de las nanoestructuras y complementar y validar los resultados de los estudios experimentales. Es un laboratorio dedicado a la síntesis, caracterización, y usos de materiales nanoestructurados semiconductores y metálicos.

\section{Universidad de Guadalajara [32]}

\section{A. Centro Universitario Valles [33]}

Aquí se realizan investigaciones relacionadas con los materiales en dos cuerpos académicos. Algunas de las líneas de investigación que se realizan son las siguientes: 
1) síntesis y propiedades de nanomateriales a base de plata y oro para aplicaciones tecnológicas: vidrios termocrómicos, filtros de luz, sensores ópticos y resistivos, colectores solares de vidrio, baterías, productos germicidas, catalizadores, etc; 2) modelación de propiedades ópticas de nanopartículas metálicas esféricas y deformadas con y sin una cáscara dieléctrica; 3 ) investigaciones de reactividad química en nanocavidades de geles de óxido de silicio; 4) diseño, síntesis y aplicaciones de materiales meso y nano estructurado; 5) estudio y aplicaciones de nano partículas metálicas soportadas en materiales nano estructurados; 6) nanoestructuras semiconductoras y nanofotónica, 7) síntesis química de partículas metálicas coloidales para la obtención de recubrimientos; 8) diseño de sistemas fotocatalíticos con alta actividad para aplicaciones ambientales; 9) estudio de materiales nanoestructurados con propiedades de centelleo para su uso en detectores de radiación; 10) investigación y desarrollo de aceleradores de partículas por técnicas electrostáticas, 11) diseño y construcción de detectores de partículas utilizando fibras ópticas con dopantes nanoestructurados.

\section{B. Centro Universitario de los Lagos [34]}

Existen dos cuerpos académicos dedicados a la investigación en nanomateriales.

En el Cuerpo Académico de Nanotecnología de Materiales se tienen las siguientes líneas de investigación: 1) espectroscopía de aniquilación de positrones para la determinación de nanoporosidades de materiales; 2) diseño, síntesis y caracterización de nanomateriales ópticos.

En el Cuerpo Académico de Bionanotecnologia las líneas de investigación son: 1) diseño, síntesis, caracterización y aplicación de biomateriales de nanocápsulas con biomoléculas de nanocompositos con estructura quiral; 2) nuevos materiales nanoestructurados con aplicaciones biomédicas.

Además, en este centro universitario se cuenta con un doctorado en ciencia y tecnología con orientación en nanotecnología y cada año se realiza un encuentro internacional de nanotecnología que abarca los topicos siguientes: nanotubos de carbón y otras nanoestructuras, nanotecnología en medicina; nanofósforos, bionanotecnología; nanoestruturas metálicas y toxicidad de nanomateriales

\section{Universidad Autónoma de San Luis Potosí (San Luis Potosi) [35]}

\section{A. Instituto de Física [36]}

Algunas de las líneas de investigación relacionadas con materiales nanoestructurados que se realizan en el Instituto de Física son las siguientes: el estudio de las propiedades electrónicas de sistemas complejos: cúmulos o agregados de átomos, superficies, defectos, multicapas, etc. (de metales de transición y semiconductores). Los problemas específicos que actualmente están bajo estudio son : determinación de la anisotropía magnética en sistemas de metales de transición de baja dimensionalidad (esta propiedad es fundamental en los dispositivos de grabación magnética); estudio de estructuras en cúmulos de metales de transición y semiconductores; propiedades electrónicas de fullerenos; aplicaciones de la mecánica estadística a sistemas como : aleaciones, sistemas magnéticos y polímeros; propiedades geométricas y catáliticas de nanoestructuras; magnetismo en cúmulos nanoestructurados, en cúmulos embebidos en sólidos; propiedades magnéticas de cúmulos de metales de transición a tem- 
peratura finita en alambres y alambres localmente adelgazados a punto de romperse; almacenamiento de gases en nanoestructuras de carbono; fabricación y estudio de materiales magnéticos nanoestructurados; efecto de las correlaciones electrónicas en las propiedades magnéticas de sistemas de baja dimensionalidad.

\section{B. Instituto de Investigación en Comunicación Óptica (IICO) [37]}

En el IICO se estudian propiedades ópticas de semiconductores, tecnología de semiconductores y dispositivos, síntesis y caracterización de materiales nanoestructurados, dispositivos orgánicos electroactivos y celdas solares, sistemas y detectores infrarrojos, nanotecnologia, MEMS, física de semiconductores, espectroscopia de semiconductores en el lejano infrarrojo, difracción de rayos x de alta resolución y, tecnología de láseres semiconductores.

\section{Facultad de Ciencias [38]}

En la Facultad de Ciencias se hace investigación en nanomateriales en el Cuerpo Académcio de Ciencia de Materiales. Allí se tienen laboratorios en modelación molecular, química de polímeros, química de materiales, espectroscopías, mecánica fina, microscopia electrónica, difracción de rayos x, metalografía, análisis térmico, pruebas mecánicas, corrosión y metalurgia de polvos.

Algunas de las líneas de investigación relacionadas con los materiales son: diseño y preparación de nanopartículas funcionalizadas; caracterización: de tamaño y forma por TEM, SEM, AFM, propiedades químicas/cristalinas por FT-IR, NMR, XRD, propiedades superficiales por BET, XPS, AFM, estructura de nanocompuestos por TEM, EDX; integración: polímeros, textiles, pinturas; dinámica de nanopartículas; escalamiento de métodos de producción; manejo de nanopartículas; propiedades biocidas de nanopartículas metálicas; cito-toxicología de nanopartículas; dispersión y consolidación.

\section{Universidad Autónoma Metropolitana (Distrito Federal) [39]}

\section{A. Departamento de Física [40]}

Algunos de los proyectos que se desarrollan en nanomateriales son: 1) propiedades magneto-ópticas en sólidos; 2) espectroscopía Raman en sólidos; 3) estudio teórico de propiedades de transporte electrónico cuántico en nanoestructuras balísticas; 4) daños por irradiación y propiedades termolumuniscentes en sólidos; 5) estudio de las propiedades termoluminiscentes de sólidos cristalinos y su aplicación a la dosimetría de la radiación ionizante; 6) óptica no lineal en vapores atómicos y en sólidos y, 7) técnicas espectroscópicas

\section{B. Laboratorio de Nanotecnología e Ingeniería Molecular [41]}

Creado inicialmente en colaboración con el Instituto Mexicano del Petróleo para mejorar el mantenimiento de los tubos de acero de Pemex, hace actualmente investigación en temas como la visualización y caracterización de moléculas de colesterol en sustratos sólidos con microscopía de fuerza atómica (AFM) y microscopía electrónica de barrido de efecto túnel (STM). 


\section{Universidad Autónoma de Morelos (Cuernavaca) [42]}

Algunas de las investigaciones que se realizan en la Universidad Autónoma de Morelos, relacionadas con los nanomateriales, son las siguientes: estados electrónicos en nitruros semiconductores: superficies, heteroestructuras simples y dobles; fonones en heteroestructuras semiconductoras basadas en nitruros; interacción electrón-fonón; estados electrónicos en sistemas delta-dopados tipo-p; estados electrónicos en heteroestructuras semiconductoras con presión hidrostática aplicada; propiedades ópticas en heteroestructuras dieléctricas cuasirregulares (Fibonacci, Thue-Morse, Period Doubling).

Estructura electrónica y fonónica de sistemas a capas (pozos cuánticos, superredes, estructuras cuasirregulares, etc.); tiempos de tunelaje de distintas excitaciones elementales (electrones, fonones, etc.) en sistemas a capas.

Teoría y simulación cuántica de materiales a escala atómico-molecular: 1) desarrollo e implementación computacional de métodos para materiales cristalinos; 2) aplicaciones sobre: a) materiales porosos, b) superficies de óxidos, c) materiales ferroeléctricos, d) minerales y e) polímeros dopados.

\section{Universidad de Sonora (Hermosillo, Sonora) [43]}

\section{A. Centro de investigación en Física (CIFUS) [44]}

En el CIFUS se desarrolla investigación científica en grupos llamados academias. En la Academia de Estado Sólido existen varias líneas de investigación relacionadas con los nanomateriales: dosimetría termoluminiscente y no termoluminiscente de halogenuros alcalinos dopados con iones de $\mathrm{Eu}^{2+}$; mecanismos de formación de daños en halogenuros alcalinos; espectroscopía de materiales aislantes dopados con impurezas divalentes $\left(\mathrm{Eu}^{2+}, \mathrm{Sn}^{2+}, \mathrm{Sr}^{2+}\right.$, etc.); vidrios y cerámicas; fabricación de materiales naestructurados ópticamente funcionales; transporte en películas delgadas semiconductoras; fabricación y caracterización de películas delgadas semiconductoras de amplio gap; nanoestructuras semiconductoras; percolación eléctrica en películas policristalinas; caracterización de materiales por técnicas fotoacústicas; instrumentación electrónica.

En la Academia de Física-Matemática se estudian sistemas de baja dimensionalidad.

En la Academia de Óptica se tienen las siguiente líneas de investigación: óptica no lineal: materiales fotorrefractivos; microscopía óptica coherente; rugosidades coherentes periódicas y aleatorias en películas delgadas.

\section{Instituto Mexicano del Petróleo (IMP) (Distrito Federal) [45]}

El IMP, creado el 23 de agosto de 1965, es el centro de investigación de México dedicado al área petrolera. Con el fin de responder a las necesidades de investigación y desarrollo tecnológico de la industria petrolera del país, el IMP cuenta con varios programas de investigación, en los que destacados científicos se ocupan de la investigación básica y su aplicación en la industria en áreas como administración del desarrollo en aguas profundas; estudio integral de yacimientos; aseguramiento de flujo; 
evaluación de cuencas/plays; control de agua; combustibles limpios; procesamiento de crudo pesado; evaluación integral de ecosistemas; evaluación integral de riesgos e ingeniería concurrente. Dentro de sus programas de investigación, el de ingeniería molecular desarrolla investigación en nanociencias y nanotecnología. El programa diseña, sintetiza y caracteriza nuevas moléculas para el desarrollo de materiales, que tengan impacto en tecnologías para el mantenimiento de flujo, recuperación secundaria y mejorada, refinación y ambiente y seguridad. Para lo cual cuenta con laboratorios de supercómputo, síntesis química y caracterización molecular con equipos modernos, e incorpora y desarrolla las teorías necesarias para el diseño molecular. Algunas de sus líneas de investigación son 1) diseño, síntesis y desarrollo de nanomateriales (catalizadores, anticorrosivos, antiespumantes, detergentes, recubrimientos); 2) mecanismos de adsorción, agregación y dispersión de coloides (mantenimiento de flujo, recuperación secundaria y mejorada, fluidos de perforación); 3) fisicoquímica de mezclas y soluciones (endulzamiento de gas, separación de nitrógeno y limpieza de lodos).

En el IMP se tiene el Laboratorio de Microscopía Electrónica de Ultra Alta Resolución, su objetivo es la caracterización estructural volumétrica química y de superficie a nivel micrométrico, nanométrico y atómico de materiales para su diseño y utilización en el desarrollo de tecnologías y productos con alto valor para la industria petrolera. Los materiales nanoestructurados diseñados para su aplicación en la industria petrolera requieren de métodos de caracterización con alta resolución espacial para determinar su forma, composición y estructura cristalina logrando así una base objetiva para el entendimiento, diseño y optimización de sus propiedades. La caracterización de dispositivos con componentes nanométricos y micrométricos, también requiere de alta resolución en tareas de observación no destructiva y de control de calidad.

La caracterización estructural es de gran importancia en la producción y aplicación de nanoestructuras ya que es indispensable para entender, diseñar y optimizar sus propiedades. La caracterización a través de microscopía electrónica ocupa un lugar preponderante dentro de las técnicas disponibles y ofrece una alta resolución espacial. Los materiales nanoestructurados están conformados por nanobloques con al menos una dimensión por debajo de $100 \mathrm{~nm}$ por lo que la alta resolución espacial adquiere un significado esencial en los procedimientos de caracterización.

Las técnicas de microscopía requieren de inversiones cuantiosas pero constituyen sin duda el método más poderoso de caracterización nanoestructural. El IMP cuenta con personal experimentado en las diferentes técnicas de caracterización microscópica, lo que permitirá lograr beneficios en la investigación, educación y generación de nuevas tecnologías.

El poder de resolución de los equipos es muy alto debido al uso de electrones que pueden manipularse para obtener imágenes de la superficie (microscopía electrónica de barrido o MEB) o de todo el espesor de la muestra (microscopía electrónica de transmisión, MET o de transmisión-barrido, STEM). Empleada adecuadamente, la microscopía electrónica permite obtener imágenes en un rango amplio que va desde características en el nivel micro hasta columnas de átomos.

Por otro lado, la microscopía electrónica ofrece la posibilidad de caracterizar la composición química de materiales y la determinación de su estructura cristalina. En 
el Laboratorio de Microscopía Electrónica de Ultra Alta Resolución del IMP se cuenta con los aditamentos necesarios (detectores, software, etc.) para dotar a los investigadores usuarios con la posibilidad de caracterizar materiales tanto química como estructuralmente.

\section{Instituto Nacional de Investigaciones Nucleares (ININ) (Ocoyoacac, Estado de MÉxico) [46]}

Aunque el objetivo principal del Instituto ININ es realizar investigación y desarrollo en el área de la ciencia y tecnología nucleares, en sus diferentes departamentos se hacen investigaciones en nanomateriales.

En los departamentos de Tecnología de Materiales, de Física y de Estudios del Ambiente, se sintetizan y caracterizan diferentes nanomateriales. En el Departamento de Materiales Radiactivos se buscan aplicaciones de las nanopartículas.

Líneas de investigación:

A) Desarrollo de nuevos nanomateriales y su caracterización. Simulación molecular. Se tienen los siguientes proyectos: 1) métodos de síntesis de nanopartículas metálicas vía química coloidal; 2) desarrollo de materiales catalíticos a base de $\mathrm{Cu}, \mathrm{Ni}, \mathrm{Pd}, \mathrm{Pt}, \mathrm{Au}$ y Ag, soportados en óxidos mixtos, tales como $\mathrm{ZrO}_{2}-\mathrm{TiO}_{2}$, $\mathrm{ZrO}_{2}-\mathrm{CeO}_{2}$; 3); manocompositos de nanopartículas en matrices poliméricas, de haluros alcalinos y de vidrios de $\left.\mathrm{SiO}_{2} ; 4\right)$ modificación de superficies de las nanoestructuras en 0 y 1 dimensión; 4) estudio de estructuras metálicas unidimensionales sintetizadas por química coloidal y sol-gel; 5) estudio de catalizadores para aplicarlos en la generación de combustible alterno $\left(\mathrm{H}_{2}\right)$ como fuente de energía limpia, determinando su actividad catalítica.

B) Preparación de películas delgadas nanoestructuradas, síntesis de nanopartículas por ablación láser en líquidos. Se realizan estudios de las condiciones de depósito que permitan obtener películas delgadas nanoestructuradas con las propiedades físicas deseadas: composición, fase, estructura, textura, entre otras, para aplicaciones potenciales como: fotoelectrodos, sensores, cátodo en microbaterías, recubrimientos duros, tribológicos, luminiscentes, etc. También se realiza síntesis de nanopartículas metálicas (Au y Ag) con diferente tamaño para modificar propiedades eléctricas de compositos poliméricos

C) Desarrollo de tecnologías de plasma y nanotecnología para aplicaciones ambientales. Se realiza síntesis de nanoestructuras de carbono para su uso como catalizadores en el tratamiento de gases tóxicos

D) Radiofármacos para diagnóstico y terapia de blancos moleculares específicos como sistemas multifuncionales utilizando nanopartículas. Se trabaja en los siguientes proyectos: 1) diseño, síntesis, caracterización fisicoquímica y evaluación de reconocimiento molecular de nanopartículas de oro conjugadas a péptidos como nanofármacos potenciales en terapia y diagnóstico; 2) funcionalizar nanopartículas de oro con péptidos para incrementar su estabilidad y biocompatibilidad permitiéndoles dirigirse a blancos moleculares específicos 


\section{Instituto Nacional de Astrofísica Óptica y Electrónica (INAOE) (Tonanzintla, Puebla) [47]}

\section{Laboratorio Nacional de Nanoelectrónica}

En abril de 2010, en el INAOE de Puebla, se inauguró el laboratorio nacional de nanoelectrónica (LNN). El objetivo de este proyecto es el de contar con un laboratorio para el desarrollo de tecnología nacional para la fabricación de dispositivos, circuitos integrados y sistemas microelectromecánicos (MEMS), que tengan posibilidades de aplicación en la industria mediante la incorporación de materiales nanoestructurados a la tecnología del silicio. El LNN está planeado para formar recursos humanos altamente calificados, con una visión moderna y global de la electrónica. El laboratorio actuará como enlace entre la investigación de alto nivel tecnológico y el sector industrial. Se espera que el LNN sea el aglutinador de los esfuerzos de todas las instituciones y centros de investigación nacionales que realizan investigación en electrónica y áreas relacionadas. El objetivo general será combinar la investigación básica y aplicada en un amplio rango de dominios de investigación, que van desde el diseño de circuitos integrados, pasando por las tecnologías de fabricación hasta microsistemas y nuevas técnicas de fabricación de circuitos impresos.

Entre los objetivos específicos se pueden nombrar: el desarrollo de una tecnología nacional de fabricación de MEMS y sistemas nanoelectromecánicos (NEMS). Fabricación de dispositivos avanzados (por ejemplo, de múltiple compuerta, FinFET) en sustratos tanto convencionales como de alto índice de refracción. Desarrollo de dispositivos optoelectrónicos en base de silicio. Realización de nuevas técnicas de diseño para la óptima utilización de los dispositivos y materiales que se desarrollan con énfasis en circuitos integrados de RF y optoelectrónicos.

\section{Instituto Potosino de Investigación Científica y Tecnológica (IPICYT) (SAN LuIs Potosí) [48]}

En la División de Materiales Avanzados del IPICyT se realizan investigaciones teóricas y experimentales de nuevos materiales tales como materiales nanoestructurados, materiales para su aplicación en electrónica orgánica. Esto con el objetivo de entender sus propiedades mecánicas, electrónicas y magnéticas, y con esta información poderlos aplicar en tecnologías novedosas. En particular, se desarrolla investigación acerca de nanoestructuras de carbono, este tema se trata en un capítulo especial. En esta división se desarrollan estudios en los campos de la nanociencia y la nanotecnología. Las líneas de investigación de la división se pueden resumir en las siguientes: estructura atómica de materiales complejos; nuevos materiales nanoestructurados; propiedades magnéticas de nuevos materiales nanoestructurados; obtención y caracterización de materiales magnéticos; polvos y láminas delgadas; magnetometría vibracional; propiedades magnéticas en sistemas de baja dimensionalidad; superficies de aleaciones metálicas; y dinámica molecular.

Dentro del IPICYT se estableció el Laboratorio Nacional de Investigaciones en Nanociencias y Nanotecnología (LINAN). Siendo uno de sus objetivos principales ser un laboratorio de acceso nacional para los institutos de investigación, las universidades nacionales y del estado, los institutos tecnológicos y las industrias en la región central 
del país, con el propósito de impulsar la investigación y la tecnología enfocada a la nanotecnología en México. El LINAN promueve las siguientes áreas de investigación en nanociencia y nanotecnología: síntesis de nanoestructuras y biomimetics; nanomateriales magnéticos y sus aplicaciones; nanobiotecnología; caracterización de biomateriales nanoestructurados; nanocompuestos; cálculos electrónicos de nanosistemas.

\section{Centro de Investigación en Materiales Avanzados S. C. (CIMAV) (Chinuahua) [49]}

La investigación en el CIMAV se realiza en tres grandes áreas: física de materiales, química de materiales y, medio ambiente y energía. Dentro del CIMAV se encuentra el Laboratorio Nacional de Nanotecnología, el cual representa una avanzada plataforma tecnológica para el impulso de la nanociencia y la nanotecnología en México, apoyando a los sectores académico, productivo y social.

Algunas de las líneas de investigación que se realizan en el CIMAV relacionadas con nanomateriales son: 1) nanomagnetismo: fluidos magnéticos de magnetita y ferrita de cobalto, fluidos magnetoreológicos de Fe-Y-Sm-Co, compuestos de Mn y tierras raras con efecto magnetocalórico; 2) superconductividad en compuestos con Fe-Se, Fe-Te, Fe-As-La-F-0; 3) materiales multiferroicos: Fe-Bi-0, PZT y ferrita de cobalto; 4) aleado mecánico: aleaciones base aluminio; 5) deterioro de materiales: aceros al carbono, aceros inoxidables, superaleaciones, aleaciones de cobre y aleaciones de aluminio, cemento; 6) recubrimientos nanoestructurados: óxidos, carburos, nitruros, titanatos y vanadatos, refractarios, recubrimientos con tierras raras; 7) polímeros nanoestructurados: quantum dots, polímero-CNT, polímero-nanopartículas, polímero-nanoarcillas, polímeros conductores; 8) catalizadores funcionalizados: zeolitas, nanopartículas metálicas, óxidos nanoestructurados, nanotubos funcionalizados, carbón activado; 9) sondas moleculares: ADN, proteínas, fulerenos y nanotubos funcionalizados, colorantes; 10) modelado molecular: colorantes, óxidos metálicos nanoestructurados, electrolitos, polímeros conductores, fulerenos y nanotubos funcionalizados, nanopartículas, psoralenos, drogas fotosensibilizadoras; 11) simulación de clusters nanométricos: óxido de litio manganeso dopado con metales de transición, nanofibras de óxido de zinc dopadas con metales de transición; 12) materiales para almacenamiento y generación de energía: tamices moleculares, nanotubos de carbón, óxidos metálicos semiconductores, óxidos no metálicos.

\section{Centro de Investigaciones en Óptica (León, Guanajuato) [50]}

El Grupo de Propiedades Ópticas de Nano-Sistemas, Interfaces y Superficies, del Centro de Investigaciones en Óptica, se ha dedicado al cálculo teórico de propiedades ópticas de superficies, interfaces y sistemas nanoscópicos. Se estudia tanto la respuesta óptica lineal como la respuesta óptica no lineal con gran éxito a través de espectroscopías ópticas de superficie. El grupo cuenta con veinte años trabajando en el tema, el cual ha ido evolucionando tanto en complejidad de los cálculos como en los sistemas en los que trabaja.

Por otra parte, otro grupo de investigadores se ha dedicado a la preparación y caracterización de materiales nanoestructurados, nanopartículas dieléctricas, semiconductoras y metálicas para utilizarlas en aplicaciones de dispositivos fotónicas como 
son: emisión de luz visible mediante excitación UV, Azul e IR para aplicaciones en desplegados y fuentes de luz, sensores ópticos, dosímetros y centelladores. Este grupo en particular organiza el congreso "Nanotech" que se ha realizado en diferentes sedes y cuyo objetivo es ser un foro que promueva la colaboración entre diferentes grupos tanto nacionales como internacionales. Los tópicos que abarca dicha conferencia son: 1. Nanotubos de carbón y otros; 2. Metales nanoestructurados; 3. Nanoestructras magnéticas; 4 . Aplicacion de nanoestructuras en celdas de combustible, catalisis y celdas solares; 5 . Nanofósforos: óxidos, puntos cuánticos y silicio poroso; 6 . Propiedades ópticas lineales y no lineales de nanocristales; 7. Nanometrología; 8. Aplicaciones médicas de nanotecnología; 9. Cristales fotónicos.

Finalmente, en el CIO también se está desarrollando un proyecto en colaboración con el Instituto de Fisica de la Universidad de Guanajuato, consistente en la síntesis de nanocristales coloidades de semiconductores, como son los puntos cuánticos de selenuro de cadmio (CdSe).

\section{Instituto TeCnológico de Monterrey (Monterrey) [51]}

El Instituto Tecnológico de Monterrey ha creado cátedras donde se desarrolla la investigación; son grupos apoyados por fondos semilla del mismo Tecnológico. Con ellas, se busca incentivar el desarrollo de la investigación en áreas prioritarias y elevar la calidad de los programas académicos. Para llevar a cabo sus proyectos de investigación, las cátedras complementan los fondos semilla con recursos externos obtenidos de empresas y organismos públicos nacionales e internacionales.

Actualmente, el Instituto Tecnológico cuenta con 122 cátedras de investigación. Un área estratégica del Instituto vinculada con la nanociencia y la nanotecnología es la ingeniería y diseño de materiales. Dentro de ella se tiene un proyecto sobre tratamientos térmicos asistidos por plasma. El desarrollo de técnicas y procesos asistidos por plasmas para la síntesis de nuevos materiales o materiales convencionales atañe diversas áreas o disciplinas. Por medio de la síntesis de materiales se pueden generar productos convencionales: la nitruración de aceros, los recubrimientos cerámicos avanzados, la formación de diamante, entre otros. Por otro lado, nuevos materiales como la síntesis de fulerenos, la formación de nitruros de aluminio, nitruro de boro o los nanotubos de carbón son ejemplos de materiales que se pueden obtener por estos medios.

\section{LISTADO Y DIRECTORIO ELECTRÓNICO DE UNIVERSIDADES, CENTROS, INSTITUTOS Y DEPENDENCIAS}

[1] Universidad Nacional Autónoma de México <www.unam.mx>

[2] Centro de Nanociencias y Nanotecnología <www.cnyn.unam.mx>

[3] Instituto de Física <www.fisica.unam.mx>

[4] Instituto de Biotecnología <www.ibt.unam.mx>

[5] Instituto de Ciencias Físicas <www.fis.unam.mx>

[6] Instituto de Investigaciones en Materiales <www.iim.unam.mx>

[7] Centro de Investigaciones en Energía <www.cie.unam.mx>

[8] Instituto de Química <www.iquimica.unam.mx>

[9] Centro de Centro de Ciencias Aplicadas y Desarrollo Tecnológico $<$ www.cinstrum.unam.mx> 
[10] entro de Física Aplicada y Tecnología Avanzada <www.fata.unam.mx>

[11] Facultad de Ciencias <www.fciencias.unam.mx>

[12] Facultad de Química <www.quimica.unam.mx>

[13] Instituto Politécnico Nacional <www.ipn.mx>

[14] Centro de Nanociencia y Micro-Nanotecnología

[15] Escuela Superior de Física y Matemáticas

[16] Centro de Investigación y de Estudios Avanzados del IPN <www.cinvestav.mx>

[17] Departamento de Física <www.fis.cinvestav.mx>

[18] Sección de Física del Estado Sólido <sfes.fis.cinvestav.mx>

[19] Laboratorio de Nanoestructuras Semiconductoras <nanosem.fis.cinvestav.mx>

[20] Departamento de Física Aplicada <www.mda.cinvestav.mx>

[21] Unidad Querétaro <www.qro.cinvestav.mx>

[22] Deparatamento de Ingeniería Eléctrica <www.ie.cinvestav.mx>

[23] Sección de Electrónica del Estado Sólido <www.sees.cinvestav.mx>

[24] Benemérita Universidad Autónoma de Puebla <www.buap.mx>

[25] Centro de Investigación en Dispositivos Semiconductores <www.cids.buap.mx>

[26] Instituto de Física 'Luis Rivera Terrazas' <www.ifuap.buap.mx>

[27] Facultad de Ciencias Físico-Matemáticas <www.fcfm.buap.mx>

[28] Centro Universitario de Vinculación <www.cuv.buap.mx>

[29] Universidad Autónoma de Nuevo León <www.uanl.mx>

[30] Centro de Innovación, Investigación y Desarrollo en Ingeniería y Tecnología $<$ www.ciidit.uanl.mx>

[31] Facultad de Ciencias Físico Matemáticas <www.fcmf.uanl.mx>

[32] Universidad de Guadalajara <www.udg.mx>

[33] Centro Universitario Valles <www.cuvalles.udg.mx>

[34] Centro Universitario de los Lagos <www.lagos.udg.mx>

[35] Universidad Autónoma de San Luis Potosí <www.uaslp.mx>

[36] Instituto de Física <www.ifisica.uaslp.mx>

[37] Instituto de Investigación en Comunicación Óptica <www.iico.uaslp.mx>

[38] Facultad de Ciencias <www.fc.uaslp.mx>

[39] Universidad Autónoma Metropolitana <www.uam.mx>

[40] Departamenteo de Física <abaco.izt.uam.mx>

[41] Laboratorio de Nanotecnología e Ingeniería Molecular $<$ http://www.nikolabatina.com.mx/>

[42] Universidad Autónoma de Morelos <www.uaem.mx>

[43] Universidad de Sonora <www.uson.mx>

[44] Centro de Investigación en Física <www.cifus.uson.mx>

[45] Instituto Mexicano del Petróleo <www.imp.mx>

[46] Instituto Nacional de Investigaciones Nucleares <www.inin.mx>

[47] Instituto Nacional de Astrofísica Óptica y Electrónica <www.inaoe.mx>

[48] Instituto Potosino de Investigación Científica y Tecnológica <www.ipicyt.edu.mx>

[49] Centro de Investigación en Materiales Avanzados S.C. <www.cimav.edu.mx>

[50] Centro de Investigaciones en Óptica <www.cio.mx>

[51] Instituto Tecnológico de Monterrey <www.itesm.edu> 